\title{
Systematic Review and Meta-analysis of Postexposure Prophylaxis for Crimean-Congo Hemorrhagic Fever Virus among Healthcare Workers
}

\author{
Önder Ergönül, Şiran Keske, Melis Gökçe Çeldir, Illayda Arjen Kara, \\ Natalia Pshenichnaya, Gulzhan Abuova, Lucille Blumberg, Mehmet Gönen
}

We performed a systematic review and meta-analysis on the effectiveness of ribavirin use for the prevention of infection and death of healthcare workers exposed to patients with Crimean-Congo hemorrhagic fever virus (CCHFV) infection. Splashes with blood or bodily fluids (odds ratio [OR] 4.2 ), being a nurse or physician (OR 2.1), and treating patients who died from CCHFV infection (OR 3.8) were associated with healthcare workers acquiring CCHFV infection; $7 \%$ of the workers who received postexposure prophylaxis (PEP) with ribavirin and $89 \%$ of those who did not became infected. PEP with ribavirin reduced the odds of infection (OR $0.01,95 \% \mathrm{Cl} 0-0.03$ ), and ribavirin use $\leq 48$ hours after symptom onset reduced the odds of death (OR 0.03, $95 \% \mathrm{Cl} 0-0.58$ ). The odds of death increased 2.4 -fold every day without ribavirin treatment. Ribavirin should be recommended as PEP and early treatment for workers at mediumto-high risk for CCHFV infection.

\footnotetext{
Crimean-Congo hemorrhagic fever (CCHF) virus (CCHFV) is listed as a highly infectious pathogen that could cause a public health emergency (http://www.who. int/medicines/ebola-treatment/WHO-list-of-top-emergingdiseases/en/). CCHFV infection has been reported from $>30$ countries in Africa, Asia, Europe, and the Middle East $(1,2)$. CCHFV is a member of the genus Orthonairovirus in the family Nairoviridae that causes severe disease in humans; the reported case fatality rate is $3 \%-30 \%(1)$. Humans can become infected through the bites of ticks, by

Author affiliations: Koç University, Istanbul, Turkey (Ö. Ergönül, M.G. Çeldir, İ.A. Kara, M. Gönen); American Hospital, Istanbul (Ş. Keske); Rostov State Medical University, Rostov-on-Don, Russia (N. Pshenichnaya); South-Kazakhstan State Pharmaceutical Academy, Shymkent, Kazakhstan (G. Abuova); National Institute for Communicable Diseases, Johannesburg, South Africa (L. Blumberg)
}

DOI: DOI: https://doi.org/10.3201/eid2409.171709 contact with patient blood or bodily fluids, or by contact with blood or tissues from viremic livestock. The risk for human-to-human transmission of CCHFV increases in parallel with the lack of preparedness (3).

Healthcare workers need to be well prepared against the emerging threat of CCHF outbreaks. The efficacy of postexposure prophylaxis (PEP) with ribavirin for high-risk exposures to CCHFV needs clear evidence. The relatively low secondary attack rates of CCHFV and ethics constraints make controlled, prospective efficacy trials for ribavirin PEP unlikely. In the absence of efficacy studies, a thorough examination and logical extrapolation of the existing data can be useful for developing recommendations. The efficacy of PEP for healthcare workers exposed to CCHF patients might be similar to that for healthcare workers with high-risk exposures to Lassa fever patients (4). A series of cases of healthcare workers infected with CCHFV has been reported (5-10). Integration of the details on the exposures and the outcomes of the infections from these published reports could provide the opportunity to generate powerful conclusions about the risk for infection and death among healthcare workers. We described the efficacy of PEP with ribavirin for CCHFV infection and early ribavirin use in CCHF treatment.

\section{Methods}

\section{Search Strategy}

We performed a systematic review of individual participant data (IPD) and reported data by using PRISMA-IPD (Preferred Reporting Items for Systematic Reviews and MetaAnalyses for IPD) guidelines (11). We searched PubMed, Google, ProMED, and conference proceedings by using the keywords "Crimea-Congo hemorrhagic fever," "health care worker," "nosocomial," "CCHF," and "health professional." We included all published reports in peer-reviewed journals through September 3, 2017. 


\section{Definitions and Outcome of Interest}

We defined CCHFV exposure as visible contact or imperceptible contact (i.e., contact with patient aerosols) with a CCHF patient. Primary outcomes were infection with no symptoms, infection with symptoms, and death. The primary study objective was to assess the protective role of PEP and early ribavirin treatment. Early treatment was defined as treatment occurring $\leq 48$ hours after the onset of symptoms.

\section{Exposure Risk Groups}

Healthcare workers were grouped into 3 categories with respect to their risks for CCHFV infection. The high-risk group consisted of healthcare workers who were directly exposed to blood or bodily fluids of a CCHFV-infected patient, such as through a needle stick or splash. Healthcare workers in this group were categorized as being without personal protective equipment (PPE) of any sort by default because a PPE breach had occurred. The moderate-risk group comprised healthcare workers without a known direct exposure to blood or bodily fluids of a CCHFV-infected patient but either handled patients who bleed or visibly produced other body fluids or were involved in aerosolproducing procedures (e.g., intubation, bronchoscopy, and resuscitation) without wearing an N95 mask. The low- or unknown-risk group consisted of healthcare workers who cared for CCHFV-infected patients who did not actively bleed or produce bodily fluids and did not participate in aerosol-producing procedures.

\section{Inclusion and Exclusion Criteria}

In this study, we included healthcare workers who were exposed to CCHFV through a defined transmission event who did and did not receive PEP, healthcare workers with laboratory-confirmed CCHFV infections who had a detailed exposure history and were closely followed by laboratory tests for their clinical outcomes, and symptomatic healthcare workers who did and did not receive ribavirin $\leq 48$ hours after the onset of symptoms. In the reports from Albania (12,13), Pakistan $(14,15)$, South Africa $(9,16,17)$, and India $(18,19)$, some of the cases were duplicates (included in $>1$ article). In these instances, we avoided duplicated data and selected the case information from the earlier publication for inclusion. We did not include seroprevalence studies, gray literature, or screening reports for tracing cases that did not have defined exposures; if the information was incomplete or lacking, we requested the information directly from the authors, and we did not include the articles if the data we needed were unavailable.

\section{Data Collection}

We entered IPD obtained from reports into a structured data sheet. We performed analyses using an integrated dataset in Stata version 11 (https://www.stata.com/). In our dataset, we included information on demographics, transmission, PEP, the course of infection, the number of days from onset of disease, and treatment. The dataset also included information on the predictors of infection and death. Study authors (Ö.E., Ş.K., M.G.Ç., and İ.A.K.) resolved discrepancies during discussions with local physicians.

\section{Statistical Analysis}

We followed the PRISMA-IPD statement guidelines (11) using R studio (https://www.rstudio.com/). We used a 2 -stage approach for analyses. First, we analyzed the studies that were suitable for calculating an effect estimate (odds ratio [OR]). Then, we pooled all the participant studies, including single case reports, and calculated a common effect estimate (OR) and 95\% CI. We used random effects models.

\section{Bias Assessment}

We performed an analysis for confounders with the integrated dataset. We used the $\chi^{2}$ test for categorical data and $t$-test for continuous data and performed logistic regression to detect potential confounding predictors of infection and death. To predict infection, we included in our model the covariates PEP with ribavirin, being in the high-risk group, being a nurse or physician, and providing care for a CCHF patient who died. To predict death, we included in our model the covariates days from onset of symptoms to ribavirin treatment, being in the high-risk group, and being a nurse or physician. These were the most critical variables for predicting death. In statistical analyses, we used Stata version 11 , and we considered $p$ values $<0.05$ statistically significant. For meta-analysis, we used meta: General Package for Meta-Analysis version 4.9-1 (https://cran.r-project.org/ web/packages/meta/index.html).

\section{Results}

We reviewed 1,224 published reports on CCHF, and 33 studies met our inclusion criteria (Figure 1). In the included studies, 175 healthcare workers from Turkey (5,7,20-25), Pakistan (15,26-32), Germany (6), Iran (33-36), India $(18,19)$, South Africa (9), Russia (8), Tajikistan (37), United Arab Emirates (38), Mauritania (39), Kazakhstan (40), Sudan (41,42), Albania (12), and Spain (2) were exposed to patients infected with CCHFV (Table). We included all of the healthcare workers who were infected, but because of the lack of detailed exposure histories among those who were not infected, we excluded 47 healthcare workers from Tajikistan (37), 75 from Turkey $(5,10), 40$ from Germany (6), and 33 from Pakistan $(15,30)$. The diagnoses were based on reverse transcription PCR results for $58(57 \%)$ healthcare workers, ELISA for 47 (46\%), both ELISA and reverse transcription PCR 


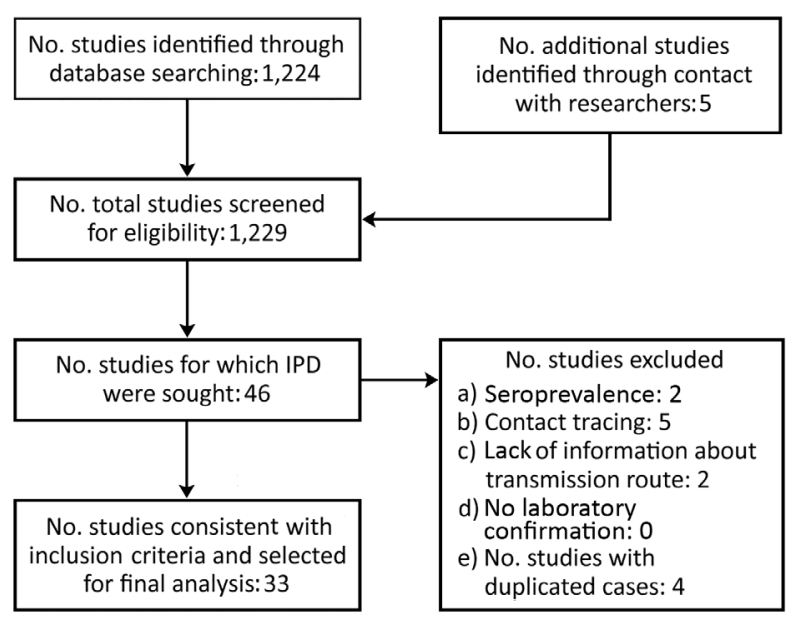

Figure 1. Identification and selection of studies included in a meta-analysis of the effectiveness of postexposure prophylaxis with ribavirin and early treatment with ribavirin among healthcare workers exposed to patients infected with CrimeanCongo hemorrhagic fever virus, 1976-2017. IPD, individual participant data.

for $26(25 \%)$, immunofluorescence assay for $13(12 \%)$, and complement fixation for $10(10 \%)$.

The population of healthcare workers included in our study was $53 \%$ male and $47 \%$ female. The percentages of infected male and female healthcare workers did not differ $(\mathrm{p}=0.828)$, and the percentage of symptomatic male $(38 \%)$ and female $(29 \%)$ healthcare workers who died was not significantly different $(\mathrm{p}=0.413)$. Among symptomatic healthcare workers, the mean age was 33 (SD 8.5, range 20-61) years, and the case-fatality rate was $34 \%$. The percentage of symptomatic cases did not differ from the percentage of asymptomatic cases $(\mathrm{p}=0.545)$, and the case-fatality rate was not higher in symptomatic than asymptomatic healthcare workers $(\mathrm{p}=0.674)$. Being a nurse or physician $(\mathrm{p}=0.01)$ and caring for a CCHF patient who died $(94 \%$ of infected healthcare workers vs. $80 \%$ of noninfected healthcare workers; $p=0.006$ ) were factors more common among healthcare workers who were infected than those who were not.

We performed 2 meta-analyses: 1 on the effectiveness of PEP with ribavirin to prevent CCHFV infection (Figure 2, panel A) and 1 on the effectiveness of early ribavirin treatment after CCHF symptom onset to prevent death (Figure 2, panel B). In the first stage of the meta-analysis on PEP, the OR could be calculated for only 4 studies (OR $0.05,95 \%$ CI $0.01-0.26$ ); at the second stage, after pooling all healthcare worker study data, the OR was 0.01 (95\% CI 0-0.03; Figure 2, panel A). The heterogeneity of these studies was not significant $\left(\mathrm{I}^{2}=3 \%, \Gamma^{2}=0.1157 ; \mathrm{p}=0.38\right)$. During the first stage of the meta-analysis on early ribavirin use, the OR could be calculated for only 2 studies (OR 0.04 , 95\% CI 0-1.33); at the second stage of the analysis, after pooling all healthcare worker study data, the OR was 0.03 (95\% CI 0-0.58; Figure 2, panel B). No heterogeneity was detected among these studies $\left(I^{2}=0 \%, \Gamma^{2}=0 ; p=0.92\right)$.

In univariate analyses of healthcare workers exposed to CCHF patients, splashes with blood or bodily fluids (OR $4.2,95 \%$ CI $2.04-9.7 ; \mathrm{p}<0.001)$, being a nurse or physician (OR 2.1, 95\% CI 1.13-4.1; $\mathrm{p}=0.019$ ), and caring for a patient who died (OR 3.8, 95\% CI 1.38-10.46; $\mathrm{p}=0.01$ ) significantly increased the odds of a healthcare worker acquiring an infection. However, PEP with ribavirin significantly reduced the risk for CCHFV infection (OR 0.01, 95\% CI 0.003-0.03; $\mathrm{p}<0.001)$. To control for confounders, we performed a multivariate analysis of the dataset. In multivariate analyses of exposed healthcare workers, PEP with ribavirin was found to significantly protect against CCHFV infection (OR 0.009, 95\% CI 0.001-0.039; p<0.001). In a sensitivity analysis, the area under the receiver operating curve was $94 \%$. In a multivariate analysis of symptomatic healthcare workers adjusted by risk group (high risk vs. others) and worker type (nurse or physician vs. others), the

Table. Characteristics and outcomes for healthcare workers exposed to patients with Crimean-Congo hemorrhagic fever virus infection, 1976-2017

\begin{tabular}{|c|c|c|c|c|c|c|}
\hline \multirow[b]{2}{*}{ Country (references) } & \multicolumn{6}{|c|}{ No. (\%) } \\
\hline & $\begin{array}{l}\text { Exposed, } \\
\mathrm{N}=175\end{array}$ & $\begin{array}{l}\text { High risk, } \\
\mathrm{n}=107\end{array}$ & $\begin{array}{l}\text { Moderate risk, } \\
\mathrm{n}=65\end{array}$ & $\begin{array}{l}\text { Low or no known } \\
\text { risk, } n=3\end{array}$ & $\begin{array}{l}\text { Infected } \\
n=102\end{array}$ & Died, $\mathrm{n}=34$ \\
\hline Turkey $(5,7,20-25)$ & $49(28)$ & $23(22)$ & $26(40)$ & 0 & $19(19)$ & $3(9)$ \\
\hline Pakistan $(15,26-32)$ & $45(26)$ & $21(20)$ & $24(36)$ & 0 & $18(18)$ & $6(18)$ \\
\hline Germany (6) & $18(10)$ & $18(17)$ & 0 & 0 & $2(2)$ & 0 \\
\hline Iran $(33-36)$ & $12(7)$ & $10(9)$ & $1(2)$ & $1(33)$ & $12(12)$ & $3(9)$ \\
\hline India $(18,19)$ & $8(5)^{\prime}$ & $5(5)$ & $3(5)$ & 0 & $8(8)$ & $6(18)$ \\
\hline Russia (8) & $8(5)$ & $6(6)$ & 0 & $2(67)$ & $8(8)$ & 0 \\
\hline South Africa (9) & $8(5)$ & $3(3)$ & $5(8)$ & 0 & $8(8)$ & $2(6)$ \\
\hline Tajikistan (37) & $7(4)$ & $7(7)$ & 0 & 0 & $7(7)$ & $2(6)$ \\
\hline United Arab Emirates (38) & $5(3)$ & $1(1)$ & $4(6)$ & 0 & $5(5)$ & $2(6)$ \\
\hline Kazakhstan (40) & $5(3)$ & $3(3)$ & $2(3)$ & 0 & $5(5)$ & $3(9)$ \\
\hline Mauritania (39) & $5(3)$ & $5(5)$ & 0 & 0 & $5(5)$ & $5(15)$ \\
\hline Sudan $(41,42)$ & $3(2)$ & $2(2)$ & $1(2)$ & 0 & $3(3)$ & $2(6)$ \\
\hline Albania (12) & $1(1)$ & $1(1)$ & 0 & 0 & $1(1)$ & 0 \\
\hline Spain (2) & $1(1)$ & $(1)^{\prime}$ & 0 & 0 & $1(1)$ & 0 \\
\hline
\end{tabular}


risk for death increased 2.4-fold for every day of delay in the start of ribavirin treatment (OR 2.4, 95\% CI 1.27-4.56; $\mathrm{p}=0.005)$. Appropriate use of PPE and PEP with ribavirin predicted death completely; therefore, both of these factors were not included in the model. The sensitivity of this model, calculated by the area under the receiver operating curve, was $95 \%$.

Of 175 healthcare workers exposed to CCHF patients, 55 (31\%) received and $110(63 \%)$ did not receive PEP with ribavirin (Figure 3). In the PEP arm, 7\% acquired infection, and in the no PEP arm, $89 \%$ acquired infection $(\mathrm{p}<0.001$; Figure 3). In the no PEP arm, 97 (99\%) of 98 infected healthcare workers became symptomatic. None of the symptomatic healthcare workers who received ribavirin within 48 hours after the onset of symptoms died, whereas $42 \%$ of the symptomatic healthcare workers who did not receive ribavirin within 48 hours died $(\mathrm{p}<0.001$; Figure 3 ). Among symptomatic healthcare workers who received ribavirin, the median time from onset of symptoms to ribavirin treatment was 5 days for those who died and 1.25 days for those who survived $(\mathrm{p}<0.001)$.

For $104(59.4 \%)$ of 175 healthcare workers, the appropriateness of the PPE was assessed by the authors of the original report. The percentage who became infected was lower for those who used PPE appropriately (55\%) than those who did not $(70 \%)$, although this difference was not significant $(p=0.301)$. No fatal cases were reported among those who used PPE appropriately. In all reports, the PPE used included a mask, gloves, and a gown; in 1 study in Germany (6), the additional use of goggles was reported.

\section{Discussion}

We analyzed all published reports on healthcare workers who were exposed to CCHF patients and had a moderateto-high risk of acquiring a CCHFV infection. These cases represent the case density in the 14 countries included, in parallel with previous reports $(43,44)$. Most cases were from Turkey, Pakistan, and Iran. However, healthcare workers could acquire the infection from persons from other countries (6), and in 2017, a nurse in Spain acquired (2) a CCH$\mathrm{FV}$ infection from a patient with an autochthonous case.

We determined that PEP with ribavirin reduced $\mathrm{CCH}$ FV infection among healthcare workers and early ribavirin use reduced death among CCHFV-infected healthcare workers (Figure 2). In most case series and case reports, no healthcare workers died who received PEP with ribavirin $(5-8,10)$, including those who received PEP soon after a high-risk incident (Figure 3).

Early use of ribavirin in the treatment of CCHFV infection has been reported as beneficial $(45,46)$ and is considered to be beneficial, despite a controversial report (47). In the report in which authors disagreed with ribavirin use being beneficial, the authors did not account for the starting time of ribavirin treatment after symptom onset,

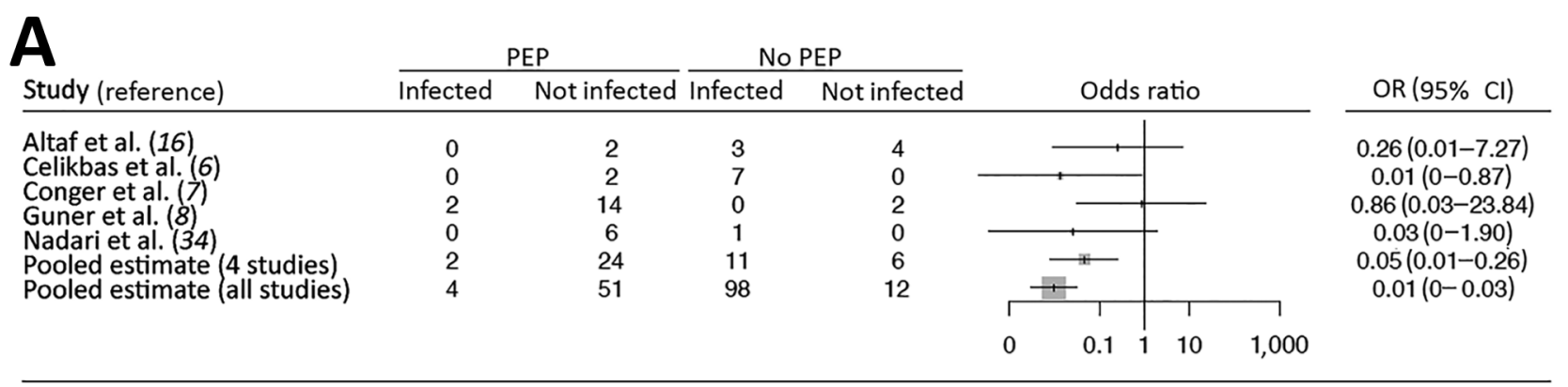

Favors PEP Favors no PEP

B

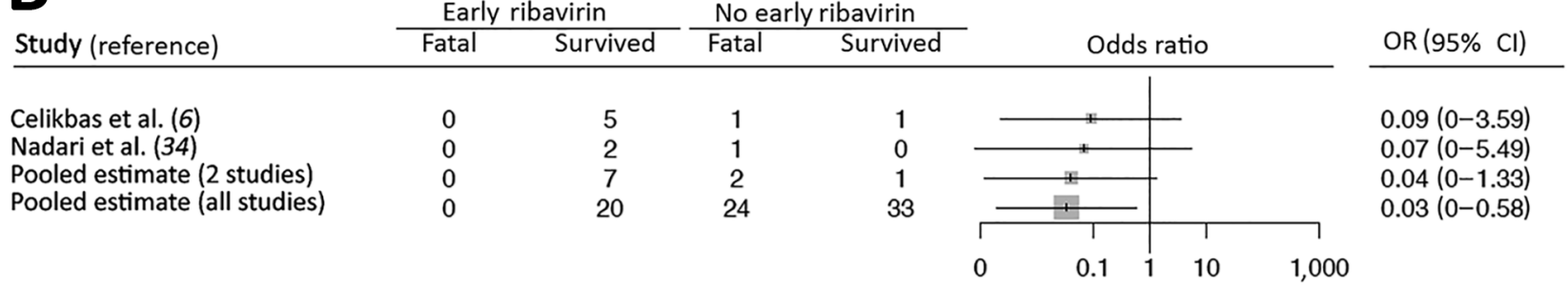

Favors early ribavirin Favors no early ribavirin

Figure 2. Effectiveness of PEP and early treatment with ribavirin among healthcare workers exposed to patients infected with CrimeanCongo hemorrhagic fever virus, 1976-2017. A) Two-step meta-analysis of the effectiveness of PEP with ribavirin for preventing Crimean-Congo hemorrhagic fever virus infection. We could determine the effect estimates for only 4 individual studies, and we included 33 reports in the final pooled estimate. B) Two-step meta-analysis on the effectiveness of early ribavirin use for preventing death caused by Crimean-Congo hemorrhagic fever virus infection. We could determine the effect estimate for only 2 individual studies, and we included 33 reports in the final pooled estimate. OR, odds ratio; PEP, postexposure prophylaxis. 


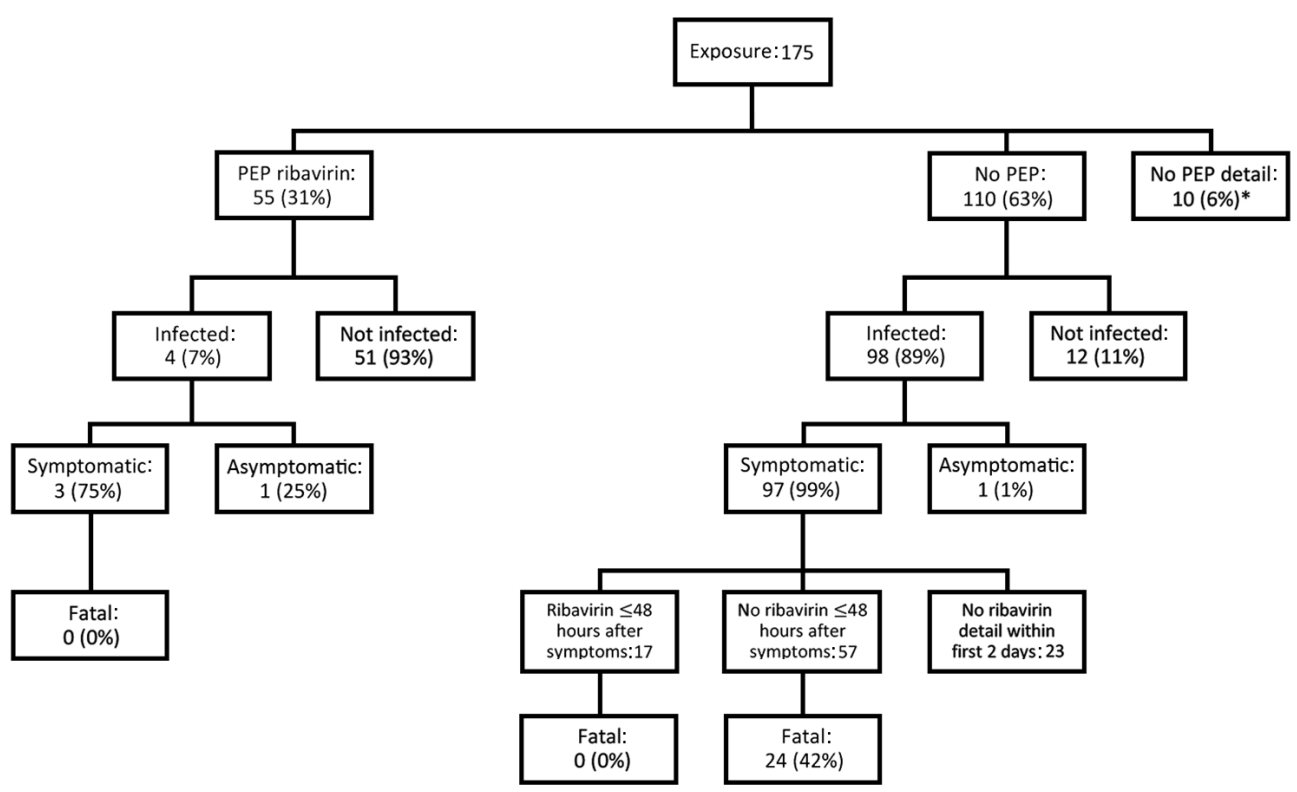

Figure 3. Flowchart of healthcare workers exposed to patients infected with Crimean-Congo hemorrhagic fever virus who did and did not receive PEP with ribavirin or early ribavirin treatment $\leq 48$ hours after symptom onset, 1976-2017. *Healthcare workers for which PEP information was not included in the original report. PEP, postexposure prophylaxis.

even though this factor can significantly alter its efficacy. Close follow-up of infected healthcare workers provides an opportunity to determine the efficacy of early ribavirin use; assessing the quality of treatments given to exposed healthcare workers is much more feasible than assessing that of patients with suspected CCHF transferred from rural areas. In this study, we showed that every day of treatment delay increases the risk for death by 2.4 -fold. Of note, none of the symptomatic healthcare workers who received ribavirin within 48 hours after the onset of symptoms died, whereas $42 \%$ of those who did not receive treatment in that time frame died $(p<0.001$; Figure 3$)$. Late diagnosis of the source case can result in delayed PEP and treatment of healthcare workers with ribavirin $(21,30)$.

Some centers have reported aerosol CCHFV transmission $(5-8,33,48)$. A number of procedures (e.g., bronchoscopy, nasal tamponade, intubation, cardiopulmonary resuscitation) as well as patient bleeding can lead to the aerosolization of CCHFV. Persons near CCHFV patients during these activities should be considered at moderate risk for acquiring the infection. Awareness of transmission after percutaneous injuries is high, but healthcare workers with imperceptible exposures to aerosolized pathogens should also be considered for close followup. Our study findings indicate that PEP with ribavirin should be recommended for those with CCHFV exposures, similar to the recommendations for healthcare workers with Lassa virus exposures (4).

In this study, we included all published reports of detailed, laboratory-confirmed cases; we avoided duplicated cases and excluded screenings of healthcare workers with nonspecified risk $(5,6,15,28,49,50)$. One limitation of this study is reporting bias; we did not include unreported cases. Because of medical and legal issues, some fatal cases involving healthcare workers who were not using PPE appropriately or who did not receive PEP might not have been reported. For example, 2 fatal cases involving healthcare workers who were not given ribavirin, 1 from Turkey (http://www.hurriyet.com.tr/gundem/kan-alirken-elineigne-batan-kubra-kkka-dan-oldu-11861967) and 1 from Pakistan (https://www.samaa.tv/uncategorized/2016/07/ congo-fever-cases-emerge-in-bahawalpur/), were not published in the literature, but their stories appeared in the media. Even though we received detailed information about these cases, we did not include them in our study because they were not published in peer-reviewed journals. These unreported fatal cases support the use of ribavirin for PEP and early treatment, as we recommend in this report.

Another limitation of this study was regarding the reporting of the appropriateness of the PPE used, which was reported for only $\approx 60 \%$ of the healthcare workers included. PPE use is not standardized; appropriate use varies substantially from country to country. For instance, in a study in Germany, the use of surgical masks instead of N95 masks during aerosol-generating procedures is considered inappropriate (6); however, this practice was considered appropriate in many other studies. Implementing standard use of PPE in healthcare settings is urgently needed. Our study shows that N95 masks should be used in high- and moderate-risk events, including those involving contact with patients who are bleeding or visibly generating bodily fluids or aerosols.

Our analyses were performed by using an integrated dataset that included all necessary detailed information about the demographics, transmission, PEP, course of the infection, days from onset of disease, and treatment. This dataset could be supplied to researchers in the field and used as a tool for future investigations. 
In conclusion, our results indicate a significant beneficial effect of PEP with ribavirin after CCHFV exposure. This beneficial effect extended to early use of ribavirin for treatment of infected healthcare workers. Imperceptible contact with infectious particles and splashes of blood or bodily fluids from infected patients should all be considered and prevented. A universal standard of care that includes PPE and PEP and treatment with ribavirin should be implemented for healthcare workers at risk for CCHF.

M.G. was supported by the Turkish Academy of Sciences (TÜBA-GEBIP, the Young Scientist Award Program) and the Science Academy of Turkey (BAGEP, the Young Scientist Award Program).

\section{About the Author}

Dr. Ergönül is professor of infectious disease and clinical microbiology at Koç University School of Medicine in Istanbul, Turkey. His primary research interests include emerging infections.

\section{References}

1. Ergönül O. Crimean-Congo haemorrhagic fever. Lancet Infect Dis. 2006;6:203-14. http://dx.doi.org/10.1016/S1473-3099(06)70435-2

2. Negredo A, de la Calle-Prieto F, Palencia-Herrejón E, Mora-Rillo M, Astray-Mochales J, Sánchez-Seco MP, et al.; Crimean Congo Hemorrhagic Fever@Madrid Working Group. Autochthonous Crimean-Congo hemorrhagic fever in Spain. N Engl J Med. 2017;377:154-61. http://dx.doi.org/10.1056/NEJMoa1615162

3. Tarantola A, Ergonul O, Tattevin P. Estimates and prevention of Crimean-Congo hemorrhagic fever risks for health-care workers. In: Ergonul O, Whitehouse CA, editors. Crimean-Congo hemorrhagic fever: a global perspective. Dordrecht (the Netherlands): Springer; 2007. p. 281-94.

4. Bausch DG, Hadi CM, Khan SH, Lertora JJ. Review of the literature and proposed guidelines for the use of oral ribavirin as postexposure prophylaxis for Lassa fever. Clin Infect Dis. 2010;51:1435-41. http://dx.doi.org/10.1086/657315

5. Celikbas AK, Dokuzoğuz B, Baykam N, Gok SE, Eroğlu MN, Midilli K, et al. Crimean-Congo hemorrhagic fever among health care workers, Turkey. Emerg Infect Dis. 2014;20:477-9. http://dx.doi.org/10.3201/eid2003.131353

6. Conger NG, Paolino KM, Osborn EC, Rusnak JM, Günther S, Pool J, et al. Health care response to CCHF in US soldier and nosocomial transmission to health care providers, Germany, 2009. Emerg Infect Dis. 2015;21:23-31. http://dx.doi.org/10.3201/ eid2101.141413

7. Guner R, Hasanoglu I, Tasyaran MA, Yapar D, Keske S, Guven T, et al. Is ribavirin prophylaxis effective for nosocomial transmission of Crimean-Congo hemorrhagic fever? Vector Borne Zoonotic Dis. 2014;14:601-5. http://dx.doi.org/10.1089/vbz.2013.1546

8. Pshenichnaya NY, Nenadskaya SA. Probable Crimean-Congo hemorrhagic fever virus transmission occurred after aerosolgenerating medical procedures in Russia: nosocomial cluster. Int J Infect Dis. 2015;33:120-2. http://dx.doi.org/10.1016/ j.ijid.2014.12.047

9. Richards GA. Nososcomial transmission of viral haemorrhagic fever in South Africa. S Afr Med J. 2015;105:709-12. http://dx.doi.org/10.7196/SAMJnew.8168

10. Leblebicioglu H, Sunbul M, Guner R, Bodur H, Bulut C, Duygu F, et al. Healthcare-associated Crimean-Congo haemorrhagic fever in Turkey, 2002-2014: a multicentre retrospective cross-sectional study. Clin Microbiol Infect. 2016;22:387.e1-4. http://dx.doi.org/ 10.1016/j.cmi.2015.11.024

11. Papa A, Bino S, Llagami A, Brahimaj B, Papadimitriou E, Pavlidou V, et al. Crimean-Congo hemorrhagic fever in Albania, 2001. Eur J Clin Microbiol Infect Dis. 2002;21:603-6. http://dx.doi.org/10.1007/s10096-002-0770-9

12. Harxhi A, Pilaca A, Delia Z, Pano K, Rezza G. Crimean-Congo hemorrhagic fever: a case of nosocomial transmission. Infection. 2005;33:295-6. http://dx.doi.org/10.1007/s15010-005-4129-x

13. Khan JA, Rehman S, Fisher-Hoch SP, Mirza S, Khurshid M, McCormick JB. Crimean Congo-haemorrhagic fever treated with oral ribavirin. Lancet. 1995;346:472-5. http://dx.doi.org/10.1016/ S0140-6736(95)91323-8

14. Altaf A, Luby S, Ahmed AJ, Zaidi N, Khan AJ, Mirza S, et al. Outbreak of Crimean-Congo haemorrhagic fever in Quetta, Pakistan: contact tracing and risk assessment. Trop Med Int Health. 1998;3:878-82. http://dx.doi.org/10.1046/ j.1365-3156.1998.00318.x

15. Swanepoel R, Shepherd AJ, Leman PA, Shepherd SP, McGillivray GM, Erasmus MJ, et al. Epidemiologic and clinical features of Crimean-Congo hemorrhagic fever in southern Africa. Am J Trop Med Hyg. 1987;36:120-32. http://dx.doi.org/10.4269/ ajtmh.1987.36.120

16. van de Wal BW, Joubert JR, van Eeden PJ, King JB. A nosocomial outbreak of Crimean-Congo haemorrhagic fever at Tygerberg Hospital. Part IV. Preventive and prophylactic measures. S Afr Med J. 1985;68:729-32.

17. Mishra AC, Mehta M, Mourya DT, Gandhi S. Crimean-Congo haemorrhagic fever in India. Lancet. 2011;378:372. http://dx.doi.org/10.1016/S0140-6736(11)60680-6

18. Patel AK, Patel KK, Mehta M, Parikh TM, Toshniwal H, Patel K. First Crimean-Congo hemorrhagic fever outbreak in India. J Assoc Physicians India. 2011;59:585-9.

19. Stewart LA, Clarke M, Rovers M, Riley RD, Simmonds M, Stewart G, et al.; PRISMA-IPD Development Group. Preferred reporting items for systematic review and meta-analyses of individual participant data. The PRISMA-IPD statement. JAMA. 2015;313:1657-65. http://dx.doi.org/10.1001/jama.2015.3656

20. Bulut C, Yilmaz GR, Karakoc E, Önde U, Kocak Tufan Z, Demiröz AP. Risk of Crimean-Congo haemorrhagic fever among healthcare workers. Presented at: 19th European Congress of Clinical Microbiology and Infectious Diseases; May 16-19, 2009; Helsinki, Finland.

21. Guven G, Talan L, Altintas ND, Memikoglu KO, Yoruk F, Azap A. An unexpected fatal CCHF case and management of exposed health care workers. Int J Infect Dis. 2017;55:118-21. http://dx.doi.org/10.1016/j.ijid.2016.12.026

22. Parlak E, Ertürk A, Koşan Z, Parlak M, Özkurt Z. A nosocomial outbreak of Crimean-Congo hemorrhagic fever. J Microbiol Infect Dis. 2015;5:5-9. http://dx.doi.org/10.5799/ ahinjs.02.2015.01.0166

23. Yildirmak T, Tulek N, Bulut C. Crimean-Congo haemorrhagic fever: transmission to visitors and healthcare workers. Infection. 2016;44:687-9. http://dx.doi.org/10.1007/s15010-016-0923-x

24. Tütüncü EE, Gurbuz Y, Ozturk B, Kuscu F, Sencan I. Crimean Congo haemorrhagic fever, precautions and ribavirin prophylaxis: a case report. Scand J Infect Dis. 2009;41:378-80. http://dx.doi.org/10.1080/00365540902882434

25. Sunbul M, Esen S, Fletcher TE, Dilek A, Guler N, Beeching NJ, et al. A fatal case of healthcare associated Crimean-Congo haemorrhagic fever with severe disease and multi-organ failure. J Infect. 2016; 72:253-5. http://dx.doi.org/10.1016/j.jinf.2015.10.005

26. Burney MI, Ghafoor A, Saleen M, Webb PA, Casals J. Nosocomial outbreak of viral hemorrhagic fever caused by Crimean Hemorrhagic fever-Congo virus in Pakistan, January 
1976. Am J Trop Med Hyg. 1980;29:941-7. http://dx.doi.org/ 10.4269/ajtmh.1980.29.941

27. Bangash SA, Khan EA. Treatment and prophylaxis with ribavirin for Crimean-Congo hemorrhagic fever-is it effective? J Pak Med Assoc. 2003;53:39-41.

28. Athar MN, Baqai HZ, Ahmad M, Khalid MA, Bashir N, Ahmad AM, et al. Short report: Crimean-Congo hemorrhagic fever outbreak in Rawalpindi, Pakistan, February 2002. Am J Trop Med Hyg. 2003;69:284-7.

29. Hasan Z, Mahmood F, Jamil B, Atkinson B, Mohammed M, Samreen A, et al. Crimean-Congo hemorrhagic fever nosocomial infection in a immunosuppressed patient, Pakistan: case report and virological investigation. J Med Virol. 2013;85:501-4. http://dx.doi.org/10.1002/jmv.23473

30. Yadav PD, Patil DY, Shete AM, Kokate P, Goyal P, Jadhav S, et al. Nosocomial infection of CCHF among health care workers in Rajasthan, India. BMC Infect Dis. 2016;16:624. http://dx.doi.org/ 10.1186/s12879-016-1971-7

31. Yadav PD, Raut CG, Mourya DT. Re-occurrence of CrimeanCongo haemorrhagic fever in Ahmedabad, Gujarat, India (2012): a fatal case report. Indian J Med Res. 2013;138:1027-8.

32. Jamil B, Hasan RS, Sarwari AR, Burton J, Hewson R, Clegg C. Crimean-Congo hemorrhagic fever: experience at a tertiary care hospital in Karachi, Pakistan. Trans R Soc Trop Med Hyg. 2005;99:577-84. http://dx.doi.org/10.1016/ j.trstmh.2005.03.002

33. Naderi HR, Sarvghad MR, Bojdy A, Hadizadeh MR, Sadeghi R, Sheybani F. Nosocomial outbreak of Crimean-Congo haemorrhagic fever. Epidemiol Infect. 2011;139:862-6. http://dx.doi.org/10.1017/ S0950268810002001

34. Naderi HR, Sheybani F, Bojdi A, Khosravi N, Mostafavi I. Fatal nosocomial spread of Crimean-Congo hemorrhagic fever with very short incubation period. Am J Trop Med Hyg. 2013;88:469-71. http://dx.doi.org/10.4269/ajtmh.2012.12-0337

35. Mardani M, Keshtkar-Jahromi M, Ataie B, Adibi P. CrimeanCongo hemorrhagic fever virus as a nosocomial pathogen in Iran. Am J Trop Med Hyg. 2009;81:675-8. http://dx.doi.org/10.4269/ ajtmh.2009.09-0051

36. Chinikar S, Shayesteh M, Khakifirouz S, Jalali T, Rasi Varaie FS, Rafigh M, et al. Nosocomial infection of Crimean-Congo haemorrhagic fever in eastern Iran: case report. Travel Med Infect Dis. 2013;11:252-5. http://dx.doi.org/10.1016/j.tmaid.2012.11.009

37. Tishkova FH, Belobrova EA, Valikhodzhaeva M, Atkinson B, Hewson R, Mullojonova M. Crimean-Congo hemorrhagic fever in Tajikistan. Vector Borne Zoonotic Dis. 2012;12:722-6. http://dx.doi.org/10.1089/vbz.2011.0769

38. Suleiman MNEH, Muscat-Baron JM, Harries JR, Satti AGO, Platt GS, Bowen ETW, et al. Congo/Crimean haemorrhagic fever in Dubai: an outbreak at the Rashid Hospital. Lancet. 1980;316:93941. http://dx.doi.org/10.1016/S0140-6736(80)92103-0

39. Nabeth P, Cheikh DO, Lo B, Faye O, Vall IO, Niang M, et al. Crimean-Congo hemorrhagic fever, Mauritania. Emerg Infect Dis. 2004;10:2143-9. http://dx.doi.org/10.3201/eid1012.040535
40. ProMED-RUS. Crimean-Congo hemorrhagic fever-Kazakhstan (South Kazakhstan). ProMed. 2009 Jul 15 [cited 2016 Feb 21]. http://www.promedmail.org/, archive no. 20090715.2529.

41. Aradaib IE, Erickson BR, Mustafa ME, Khristova ML, Saeed NS, Elageb RM, et al. Nosocomial outbreak of CrimeanCongo hemorrhagic fever, Sudan. Emerg Infect Dis. 2010;16:8379. http://dx.doi.org/10.3201/eid1605.091815

42. Elata AT, Karsany MS, Elageb RM, Hussain MA, Eltom KH, Elbashir MI, et al. A nosocomial transmission of Crimean-Congo hemorrhagic fever to an attending physician in North Kordufan, Sudan. Virol J. 2011;8:303. http://dx.doi.org/10.1186/ 1743-422X-8-303

43. Bente DA, Forrester NL, Watts DM, McAuley AJ, Whitehouse CA, Bray M. Crimean-Congo hemorrhagic fever: history, epidemiology, pathogenesis, clinical syndrome and genetic diversity. Antiviral Res. 2013;100:159-89. http://dx.doi.org/10.1016/ j.antiviral.2013.07.006

44. Ince Y, Yasa C, Metin M, Sonmez M, Meram E, Benkli B, et al. Crimean-Congo hemorrhagic fever infections reported by ProMED. Int J Infect Dis. 2014;26:44-6. http://dx.doi.org/10.1016/ j.ijid.2014.04.005

45. Dokuzoguz B, Celikbas AK, Gök SE, Baykam N, Eroglu MN, Ergönül Ö. Severity scoring index for Crimean-Congo hemorrhagic fever and the impact of ribavirin and corticosteroids on fatality. Clin Infect Dis. 2013;57:1270-4. http://dx.doi.org/10.1093/cid/ cit527

46. Ergonul O. Treatment of Crimean-Congo hemorrhagic fever. Antiviral Res. 2008;78:125-31. http://dx.doi.org/10.1016/ j.antiviral.2007.11.002

47. Ergonul O. DEBATE (see Elaldi $\mathrm{N}$ et al, Efficacy of oral ribavirin treatment in Crimean-Congo haemorrhagic fever: a quasi-experimental study from Turkey. Journal of Infection 2009; 58: 238-244): biases and misinterpretation in the assessment of the efficacy of oral ribavirin in the treatment of Crimean-Congo hemorrhagic fever. J Infect. 2009;59:284-6. http://dx.doi.org/ 10.1016/j.jinf.2009.08.006

48. Pourahmad M, Raoofi R, Chinikar S, Ghiasi SM, Ghalyanchi-Langeroudi A. Nosocomial transmission of CrimeanCongo hemorrhagic fever in a health care worker, Fars Province, Iran. Iran J Clin Infect Dis. 2011;6:47-50.

49. Ergonul O, Zeller H, Celikbas A, Dokuzoguz B. The lack of Crimean-Congo hemorrhagic fever virus antibodies in healthcare workers in an endemic region. Int J Infect Dis. 2007;11:48-51. http://dx.doi.org/10.1016/j.ijid.2005.10.009

50. Mardani M, Rahnavardi M, Rajaeinejad M, Naini KH, Chinikar S, Pourmalek F, et al. Crimean-Congo hemorrhagic fever among health care workers in Iran: a seroprevalence study in two endemic regions. Am J Trop Med Hyg. 2007;76:443-5.

Address for correspondence: Önder Ergönül, Koç University, School of Medicine, Infectious Diseases Department, Davutpaşa Caddesi, No. 4, 34010 Topkapi, Istanbul, Turkey; email: oergonul@ku.edu.tr 